Questions vives

\section{Questions Vives}

Recherches en éducation

Vol. $6 n^{\circ} 18 \mid 2012$

Les recherches sur les pratiques enseignantes efficaces

\title{
La modération sociale : un dispositif soutenant l'émergence de savoirs négociés sur l'évaluation certificative des apprentissages des élèves
}

Social moderation: A design that supports knowledge's negotiation on certificative assessment of students' learning

Lucie Mottier Lopez, Walther Tessaro, Lionel Dechamboux et Fernando Morales Villabona

\section{OpenEdition}

\section{Journals}

Édition électronique

URL : http://journals.openedition.org/questionsvives/1235

DOI : 10.4000/questionsvives. 1235

ISBN : 978-2-8218-1394-6

ISSN : 1775-433X

Éditeur

Université Aix-Marseille (AMU)

Édition imprimée

Date de publication : 15 décembre 2012

Pagination : 159-175

ISSN : 1635-4079

\section{Référence électronique}

Lucie Mottier Lopez, Walther Tessaro, Lionel Dechamboux et Fernando Morales Villabona, « La modération sociale : un dispositif soutenant l'émergence de savoirs négociés sur l'évaluation certificative des apprentissages des élèves », Questions Vives [En ligne], Vol.6 n¹8| 2012, mis en ligne le 26 mai 2014, consulté le 03 mai 2019. URL : http://journals.openedition.org/questionsvives/1235 ; DOI : 10.4000/questionsvives.1235

Ce document a été généré automatiquement le 3 mai 2019.

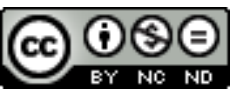

Questions Vives est mis à disposition selon les termes de la licence Creative Commons Attribution Pas d'Utilisation Commerciale - Pas de Modification 4.0 International. 


\title{
La modération sociale : un dispositif soutenant l'émergence de savoirs négociés sur l'évaluation certificative des apprentissages des élèves
}

\author{
Social moderation: A design that supports knowledge's negotiation on \\ certificative assessment of students' learning
}

Lucie Mottier Lopez, Walther Tessaro, Lionel Dechamboux et Fernando Morales Villabona

1 Après un mouvement de réformes des systèmes d'évaluation scolaire, en Suisse (Gilliéron Giroud \& Ntamakiliro, 2010) et plus largement en France, en Belgique, au Québec notamment, on constate un retour à des pratiques d'évaluation des apprentissages plus traditionnelles: renforcement des notes, des moyennes, du nombre d'étapes de certification intermédiaires à des fins de promotion ou redoublement, etc. En tant que chercheurs qui ont œuvré à promouvoir des pratiques plus innovantes, une réaction pourrait être le découragement. Pourtant, nous avons pris l'option de travailler avec les nouvelles contraintes et pratiques existantes pour mieux les comprendre et tenter de les penser dans une perspective de développement professionnel. Ainsi, la recherche que nous présentons dans cet article porte sur les pratiques d'évaluation certificative d'enseignants de l'école primaire genevoise (élèves de 8-10 ans), notamment quand ils attribuent des notes aux copies de leurs élèves qui serviront à calculer ensuite une moyenne déterminant la promotion à la fin de chaque degré scolaire. Nous centrerons notre propos sur des expériences de « modération sociale » qui amènent les enseignants à confronter leurs pratiques et à tenter de construire collectivement des savoirs partagés sur les évaluations certificatives qu'ils élaborent en français et en mathématiques.

Après une présentation des principaux enjeux que nous percevons actuellement à étudier les pratiques d'évaluation certificative des enseignants, puis de notre cadre théorique sur 
la modération sociale dans une perspective d'évaluation collaborative, l'article décrit les visées de notre recherche et son dispositif. Des premiers résultats sont exposés sur les aspects vus comme devant être harmonisés entre les évaluations certificatives des enseignants, puis donne à voir la façon dont des significations se sont socialement construites autour de l'idée d'arrangement évaluatif, offrant ainsi les conditions à l'émergence d'une distinction conceptuelle entre arrangements évaluatifs et ajustements des dispositifs d'évaluation à des fins de différenciation pédagogique.

\section{Enjeux d'une culture de l'évaluation partagée entre les enseignants}

\subsection{L'évaluation certificative des apprentissages des élèves}

3 L'analyse de la littérature des 30 dernières années en matière d'évaluation des apprentissages en classe (Mottier Lopez \& Laveault, 2008) montre que les recherches se sont essentiellement intéressées à l'évaluation formative dans sa fonction de régulation des processus d'enseignement et d'apprentissage. Ainsi, comme nous le notions :

4 Au contraire de l'évaluation formative, l'évaluation scolaire à des fins de certification héritait d'une longue histoire, pas toujours glorieuse, de mauvaises pratiques de correction, de notation et d'examens. À ceci s'ajoutaient les excès de mesures quantitatives se traduisant par une perte ou une dénaturation de l'information à cause de pratiques inadéquates de codification, de transformation des notes ou d'une mauvaise utilisation des techniques statistiques. (p. 19)

5 Récemment et à partir d'un ensemble d'études expérimentales, l'ouvrage collectif de Butera, Buchs et Darnon (2011) souligne les "menaces» potentielles produites par l'évaluation certificative sur la performance des élèves, sur leur estime de soi, sur leurs comportements psychosociaux, débouchant sur un discours de rejet de l'évaluation et des pratiques de notation. Nous pourrions suivre ces études si les politiques éducatives acceptaient de supprimer les évaluations certificatives afin de ne promouvoir que les évaluations formatives qui visent à soutenir la régulation des processus d'enseignement et d'apprentissage. Mais la réalité politique et sociale est tout autre : il est demandé aux systèmes éducatifs de certifier les acquis des élèves (par des notes et des moyennes ou, dans des systèmes plus innovants, par des appréciations et d'autres modes de communication). Cette demande de certification apparaît comme légitime compte tenu des contraintes propres aux formations post-obligatoires, à la formation professionnelle et aux exigences de l'entrée dans le métier quel qu'il soit. Le défi est alors de renforcer la qualité, la cohérence et la fiabilité des pratiques de certification des apprentissages des élèves. À nos yeux, trois enjeux sont particulièrement cruciaux dans cette perspective.

6 Le premier est de penser de façon moins cloisonnée les fonctions formative et certificative de l'évaluation scolaire. Dans les modélisations théoriques et les recommandations pratiques des premières heures, ces deux fonctions de l'évaluation sont opposées voire antinomiques: il y aurait une «bonne» évaluation, celle qui soutient l'apprentissage et fournit des informations pour décider des adaptations nécessaires aux processus d'enseignement par rapport aux caractéristiques et besoins des élèves (évaluation formative); il y aurait une « mauvaise » évaluation, celle qui classe les élèves par le moyen des notes chiffrées et qui sert un but plus ou moins avoué de sélection 
scolaire et sociale (évaluation certificative et pronostique). Persister à penser l'évaluation scolaire dans cette dichotomie conduit, à notre avis, à une impasse: celle de ne pas modéliser l'évaluation certificative dans les opportunités qu'elle peut aussi produire pour soutenir la régulation des processus d'enseignement et d'apprentissage. «Rien n'empêche a priori une évaluation certificative de jouer un rôle de support à l'apprentissage, que ce soit au niveau cognitif ou motivationnel, tout en reconnaissant évidemment sa fonction première d'attestation des apprentissages réalisés » (Mottier Lopez \& Laveault, 2008, p. 18).

7 Le deuxième enjeu est d'articuler les évaluations internes, c'est-à-dire conçues par les enseignants ou un groupe d'enseignants, et les évaluations externes produites par le système scolaire. Aujourd'hui, plusieurs instances ont choisi de développer des épreuves externes dans une double perspective: (a) pour engager une régulation au niveau du système éducatif en tant qu'outil, parmi d'autres, de pilotage, (b) pour permettre aux enseignants et aux établissements scolaires de tendre vers une certaine harmonisation des pratiques en fonction des exigences du curriculum. L'enjeu est de renforcer la complémentarité des pratiques d'évaluation des enseignants et des évaluations externes (Laveault, 2009), mais tout en respectant la logique propre à chacune de ces formes d'évaluation (Allal, 2009).

Le dernier enjeu qui nous occupe est de promouvoir des pratiques d'évaluation dans une perspective plus collaborative entre les enseignants et autres professionnels concernés par l'évaluation des apprentissages des élèves. Traditionnellement, l'enseignement est vu comme une activité autonome, qui demande des initiatives personnelles en fonction de ce qui se passe dans la spécificité du contexte de chaque classe. Attribuer des notes aux copies des élèves représente une de ces activités professionnelles qui apparaît effectuée de façon plutôt «privée » par l'enseignant (Allal \& Mottier Lopez, à paraître). Sans nier l'importance d'une dimension individuelle qui est une des caractéristiques d'une éthique de la responsabilité (Weber, 1919), nous pensons que l'enjeu est de renforcer le développement de pratiques plus collaboratives entre enseignants afin de soutenir la qualité de leurs évaluations certificatives.

9 Par ailleurs, la littérature de recherche insiste sur les « forts enjeux » (high stakes) associés à la fonction de certification de l'évaluation des apprentissages, par rapport aux parcours scolaires et de formation des élèves : voilà une raison supplémentaire pour en faire un objet de recherche et d'intervention. Notre objectif est donc d'étudier avec une grande attention les pratiques d'évaluation certificative des enseignants, telles qu'elles s'accomplissent dans la réalité de leur contexte professionnel et institutionnel, pour mieux les comprendre d'une part et, d'autre part, pour tenter d'agir sur les conditions de leur transformation potentielle dans une perspective de développement professionnel des enseignants.

\subsection{Construction d'une culture partagée de l'évaluation}

10 L'idée de culture de l'évaluation est fréquemment citée dans la littérature de recherche ainsi que dans les discours institutionnels et politiques en matière d'éducation. Il s'agit pourtant d'une notion controversée, souvent mal définie, et qui renvoie à des réalités différentes (voir Mottier Lopez, sous presse). Dans nos travaux, nous exploitons la notion de culture de l'évaluation dans une perspective d'anthropologie située, afin notamment de désigner les normes, les pratiques, les langages, les outils d'évaluation partagés par un 
groupe social donné, dans ses dimensions pré-existantes et co-construites ${ }^{1}$. Morrissette (2011) nous sensibilise à l'idée que la notion de culture suppose aussi la reconnaissance par les membres du groupe social de ce qui peut être légitimement différent entre eux. Ainsi, à partir des catégories de l'auteure, nous distinguons les consensus socialement négociés en fonction de trois niveaux de reconnaissance :

1. Des pratiques vues comme partagées par le groupe social et pouvant être communes aux membres du groupe (voire même « devant être communes » compte tenu des prescriptions règlementaires par exemple ou des pratiques négociées au sein d'un établissement scolaire) ;

2. Des pratiques vues comme socialement admises, c'est-à-dire qui regroupent des pratiques singulières et créatrices mais qui ne sont pas considérées comme devant être nécessairement partagées par le groupe; elles sont néanmoins perçues comme étant légitimes au regard des prescriptions institutionnelles, de la culture professionnelle et du cadre déontologique des enseignants ;

3. Des pratiques qui sont contestées par le groupe social, mais qui existent malgré tout en raison d'accords pragmatiques qui concilient différents positionnements et qui négocient les tensions et contradictions perçues par les enseignants dans leur contexte d'exercice professionnel (pp. 19-24).

11 Notre postulat est que c'est en soutenant la co-construction d'une culture de l'évaluation autour d'un projet commun que nous connaîtrons mieux les valeurs, normes, pratiques, significations attribuées aux évaluations certificatives construites par les enseignants. Concomitamment, c'est par ce projet commun qu'une transformation de la culture existante est susceptible de s'engager dans une perspective de développement professionnel.

\subsection{Les pratiques de modération sociale dans une perspective d'évaluation collaborative}

Dans Allal et Mottier Lopez (à paraître), nous avons dégagé deux approches pour penser une perspective collaborative de l'évaluation, en prenant appui sur le modèle général de la communauté de pratique de Wenger (1998) qui stipule que les personnes s'engagent dans des processus de négociation de significations, à propos d'actions qu'ils doivent coordonner au regard d'un projet commun et partagé. Nous ne retiendrons ici qu'une seule de ces approches qui est celle de la modération sociale qui désigne des pratiques collectives de confrontation de "jugements humains" (Linn, 1993) sur des travaux d'élèves. A partir de cas concrets, des confrontations sociales et symboliques entre participants visent à construire collectivement une représentation commune des attentes officielles traduites dans les référents externes (plan d'études, épreuves externes, directives institutionnelles notamment) et à déboucher sur des consensus à propos des pratiques d'évaluation interne des apprentissages des élèves. Plus généralement, les buts visés par la modération sociale sont d'améliorer la fiabilité, la comparabilité et la validité des évaluations certificatives et pronostiques établies par les enseignants (Harlen, 2007), ainsi que d'assurer une plus grande cohérence (un meilleur alignement) entre le curriculum prescrit, les activités didactiques en classe et les tâches évaluatives (Laveault, 2009). Maxwell (2002) définit deux fonctions principales à la modération sociale :

- for accountability, c'est-à-dire à des fins de contrôle externe et de validation officielle des résultats de l'évaluation impliquant des experts externes ; 
- for improvement, c'est-à-dire visant à développer les compétences professionnelles des enseignants à formuler des jugements d'évaluation équitables et, dans le prolongement, à contribuer à améliorer le système éducatif.

13 Dans la littérature, plusieurs recherches étudient les processus de jugement impliqués dans les modérations sociales «for improvement ", en lien notamment avec l'implantation d'un nouveau matériel curriculaire en évaluation (e.g. Wyatt-Smith, Klenowski \& Gunn, 2010). Ce matériel (objectifs principaux d'apprentissage, standards, épreuves-types, guide pour l'enseignant) soutient les échanges de modération sociale sur des échantillons de réponses d'élèves. Des résultats sont produits sur les processus de dialogue et de négociation, sur le type de connaissances convoquées par les enseignants dans leurs jugements évaluatifs, sur les différents facteurs qui contribuent à " former » l'évaluation située des enseignants. Cependant, peu d'informations sont disponibles sur les dispositifs de médiation des pratiques de modération sociale elles-mêmes: quelles sont les démarches proposées aux enseignants pour confronter leur jugement? Quels sont les configurations sociales et outils qui soutiennent la formation des consensus? Sur quelles temporalités? Dans le prolongement des thèses situationnistes fortement inspirées des travaux de Dewey, nous considérons que les «conditions-moyens" de médiation qui soutiennent les pratiques de modération sociale sont essentielles à mieux conceptualiser, car faisant partie intégrante des savoirs collectifs et du développement professionnel susceptibles de s'y développer (Mottier Lopez, 2012).

\subsection{Buts de l'article}

Le premier but est de décrire précisément le dispositif de modération sociale que nous avons conçu à la fois dans une perspective de développement professionnel et de recherche. Ce premier apport de l'article vise à partager avec les communautés scientifiques et des formateurs un dispositif possiblement réplicable dans d'autres contextes que celui de notre recherche. Cela nous permet également de penser les processus de négociation de significations et de construction de consensus dans une alternance de configurations sociales et de temporalités qui contribuent, à notre sens, pleinement à la production de savoirs collectifs. Le deuxième but de l'article est de présenter les premiers résultats sur les savoirs collectivement négociés au cours de ces pratiques de modération sociale. Nous avons distingué deux niveaux principaux de négociation : la construction de l'instrument d'évaluation (contrôle écrit ${ }^{2}$ ou autre type de tâche évaluative) ; l'appréciation du travail de l'élève sur une tâche donnée (réponses à un contrôle écrit ou production élaborée par l'élève). Ce faisant, nous élargissons l'objet de confrontation sociale et de construction de consensus comparativement aux études consultées sur la modération sociale qui, elles, se centrent essentiellement sur la notation des copies des élèves (e.g. Wyatt-Smith et al., 2010). Enfin, le dernier but de l'article est de donner à voir comment une distinction conceptuelle s'est co-construite au fil de la modération sociale qui, ainsi, est parvenue à soutenir des échanges dont la fonction épistémique a permis de dépasser une "simple " expérience vécue dans un contexte social et institutionnel donné pour offrir des conditions à un renouvellement de la conceptualisation d'un aspect de la pratique évaluative en classe. 


\section{Une recherche-formation sur les processus de jugement des enseignants dans des évaluations certificatives}

\subsection{Le contexte de la recherche-formation}

15 de l'école primaire genevoise et quatre chercheurs, les auteurs de l'article ${ }^{4}$. Les enseignants impliqués proviennent de six établissements scolaires d'un niveau socioéconomique contrasté. Ils ont une expérience d'au moins trois ans dans les degrés concernés ; ils suivent leur groupe classe dans les deux degrés consécutifs. Une diversité d'expériences professionnelles les caractérise: type de formation, nombre d'années d'expérience, parcours professionnel, notamment.

L'école primaire genevoise a vécu une réforme importante en matière d'évaluation des apprentissages entre les années 1980 et 2005, avec notamment la promotion de l'évaluation formative, l'introduction de cycles d'apprentissage et une évaluation certificative en fin de cycle seulement. Mais suite à une votation populaire en 2006, des évaluations certificatives sanctionnées par des notes sont à nouveau exigées et des moyennes par trimestre sont réintroduites dès la $5 \mathrm{P}$ HarmoS en français et en mathématiques. Désormais, les enseignants doivent attribuer une note entière sur une échelle de 1 à 6 à chaque contrôle écrit certificatif (et éventuellement à un ensemble de « petits tests »), ils doivent calculer des moyennes à la demi-note pour les deux domaines disciplinaires. En fin de 6P HarmoS, une évaluation externe, semi-standardisée, est soumise à tous les élèves du canton. Le résultat de cette évaluation externe fait partie de la certification de fin de degré. C'est donc dans ce contexte de retour à des pratiques évaluatives plus traditionnelles que nous avons pris contact auprès des directeurs d'établissement pour obtenir leur accord, puis auprès des enseignants afin de savoir s'ils étaient intéressés à participer à notre recherche.

\subsection{Le schéma général du dispositif de recherche et de formation}

Suite à plusieurs réunions préalables de concertation entre les différents acteurs, trois volets successifs ont ponctué notre dispositif de recherche. Chacun de ces volets a fait l'objet d'une étude exploratoire préalable. Les volets 1 et 3 étudiaient le «jugement-enacte » de chaque enseignant quand il corrigeait et attribuait une note aux copies de ses élèves. Pour chacun de ces deux volets, nous avons observé les enseignants, une fois en français, une fois en mathématiques. Les premiers résultats issus du volet 1 sont présentés dans Dechamboux (2012). Quant au volet 2, il proposait un dispositif de modération sociale dans un but explicite de développement professionnel associé à notre démarche de recherche scientifique. La figure 1 reprend les trois volets de façon synthétique avant une présentation plus détaillée du deuxième volet, qui nous intéresse plus spécialement dans cet article. 


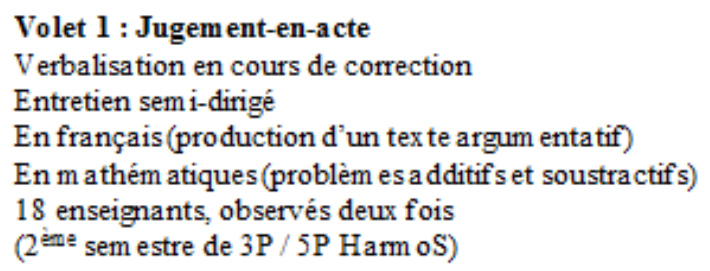

Volet 3 : Jugem ent-en-acte

Réplication du volet 1

+ Questions spécifiques au développem ent professionnel

( 2 ene sem estre de 4P/6P Harm oS)

FIgURE 1 : Les trois volets de la recherche

\section{Description du dispositif de médiation des modérations sociales}

Les modérations sociales ont été organisées sur deux jours et demi de formation continue pendant le premier trimestre de la $6 \mathrm{P}$ HarmoS. Lors des deux premières réunions (séparées d'une dizaine de jours), les participants ont travaillé en deux groupes séparés de neuf enseignants, issus pour chacun de trois établissements scolaires différents, selon un même scénario méticuleusement préparé en amont par l'équipe de recherche. La dernière demi-journée, les 18 enseignants et quatre chercheurs ont tous été réunis.

\subsection{Matériel à disposition}

19 Un ensemble de documents était préparé en amont de ces séances afin de soutenir les échanges. Des contrôles écrits, sélectionnés par les chercheurs à partir des données recueillies dans le volet 1 , ont été utilisés en proposant aux enseignants d'un groupe des copies issues de l'autre groupe, rendues anonymes (enseignant, élève et établissement). Dans un premier temps, les évaluations étaient vierges de toute réponse à des fins de confrontations sur la construction des contrôles écrits. Dans un second temps, les mêmes évaluations, mais cette fois-ci avec les réponses de l'élève, ont nourri les modérations sur l'appréciation et la notation des productions. Un matériel institutionnel était aussi à disposition des enseignants : des épreuves externes du canton de Genève dans les mêmes disciplines et domaines d'apprentissage que les évaluations internes des enseignants; le plan d'études romand (PER) nouvellement introduit en Suisse romande et que les enseignants doivent désormais utiliser ${ }^{5}$. Enfin, en amont de ces séances, les enseignants avaient été enjoints d'apporter tout autre matériel qu'ils utilisent habituellement lorsqu'ils conçoivent leurs évaluations. 


\subsection{Déroulement des modérations sociales et instrumentation des échanges} pour soutenir et structurer, sans l'enfermer, la réflexion des enseignants mais également pour conserver des traces des consensus co-élaborés. Les trios devaient se mettre d'accord afin de renseigner une grille pour chaque niveau de négociation (conception du contrôle écrit puis correction/notation). Des catégories prédéfinies et des catégories ouvertes étaient proposées aux enseignants qui devaient les croiser, pour chaque aspect de la pratique d'évaluation, aux trois types de consensus inspirés de Morrissette (2011), à savoir: les manières de faire partagées, admises ou contestées (voir le tableau 1 pour un exemple de ce type d'outil).

Tableau 1 : Exemple d'outil « Construction du contrôle écrit »

\begin{tabular}{|r|l|l|l|}
\hline Catégonies & $\begin{array}{l}\text { Manières de faire } \\
\text { partagées (reconnaissance } \\
\text { et accord collectif dans le } \\
\text { trio) }\end{array}$ & $\begin{array}{l}\text { Manières de faire } \\
\text { admises (ni adhésion, ni } \\
\text { objection dans le trio) }\end{array}$ & $\begin{array}{l}\text { Manières de faire } \\
\text { contestées (désaccords } \\
\text { persistants dans le trio) }\end{array}$ \\
\hline $\begin{array}{l}\text { Choix et fomulation des } \\
\text { objectifs }\end{array}$ & & & \\
\hline Arguments & & & \\
\hline $\begin{array}{l}\text { Points attribués à chaque } \\
\text { objectif/ critère }\end{array}$ & & & \\
\hline Arguments & & & \\
\hline $\begin{array}{l}\text { Détermination du seuil de } \\
\text { réussite }\end{array}$ & & & \\
\hline & & & \\
\hline$\ldots .$. & & & \\
\hline
\end{tabular}

À la suite de chaque temps en trio, une mise en commun était conduite par un des chercheurs qui jouait le rôle de modérateur. Ce dernier remettait en discussion les propositions des trios et les formalisait progressivement pour aboutir à un consensus au niveau de l'ensemble du groupe. Les traces de ces écritures « publiques » étaient mises en forme par l'équipe de recherche entre chaque séance de modération sociale dans un document qui était ensuite soumis systématiquement à la validation de chaque groupe pour le deuxième jour, puis en plénière lors du troisième jour. De nouveaux ajustements étaient rédigés en fonction de l'évolution des réflexions individuelles et collectives.

Des temps de réflexion individuelle furent également aménagés. À la fin de chacune des deux premières séances, les enseignants recevaient leurs propres contrôles écrits (récoltés dans le volet 1). Au regard des consensus élaborés collectivement, il leur a été demandé de mener une réflexion critique sur leur propre pratique d'évaluation. Ce retour réflexif et introspectif était instrumenté par un document ad hoc qu'ils avaient à renseigner. 


\subsection{Un processus de construction de savoirs collectifs négociés et de validité de signifiance}

L'ensemble des interactions verbales ont été enregistrées et partiellement transcrites. Toutes les traces écrites (semi-collectives, collectives et individuelles) ont été recueillies pour en faire des analyses de contenu et pour procéder à des triangulations méthodologiques.

Notre dispositif de modération sociale visait à favoriser des confrontations et la construction sociale de consensus non seulement entre les subjectivités de chaque enseignant, mais également au regard d'un sens collectif progressivement élaboré à partir des processus intersubjectifs. En ce sens, notre définition de la modération sociale, comparativement aux travaux consultés, introduit plus explicitement la co-construction de « savoirs collectifs négociés » (Morrissette, Mottier Lopez \& Tessaro, 2012) qui exige un processus itératif de formalisation des consensus (de trois types de reconnaissance: partagé, admis, contesté) dégagés par les trios, un deuxième niveau de consensus entre les trios dont les échanges étaient guidés par une modération critique du chercheur, une écriture collective des savoirs négociés, et une confrontation des savoirs formalisés dans chaque groupe puis à l'ensemble des enseignants lors de la dernière journée de formation. Ce processus itératif a pour but d'assurer une validité sociale et de signifiance (Pourtois \& Desmet, 1997) aux savoirs négociés ainsi dégagés. Ce sont ces savoirs qui ont été plus spécialement analysés pour dégager les principaux consensus qui ont émergé et qui sont présentés ci-dessous.

\section{Consensus sur des aspects vus comme devant être harmonisés entre les évaluations certificatives des enseignants}

Deux grandes catégories de consensus ont émergé à propos des éléments que le groupe a considérés comme devant être comparables entre enseignants à des fins d'harmonisation et d'équité pour les élèves. La première porte sur l'usage du plan d'études romand et la deuxième sur le champ du contrôle écrit, appelé « entête » dans le contexte genevois. On note déjà ici que ce sont donc sur des aspects qui touchent le cadre de référence de l'évaluation (le plan d'études, les objectifs d'apprentissage, les critères d'évaluation) et non pas sur des aspects plus sensibles - moins observables et formalisables - relevant des processus mêmes d'interprétation des réponses / productions des élèves.

\subsection{Usage du plan d'études (PER)}

Comme indiqué précédemment, un nouveau plan d'études est en cours d'introduction en Suisse romande et les enseignants de notre recherche l'utilisaient pour la première année. Il ressort qu'ils s'accordent à considérer que la première exigence pour tous les enseignants est un alignement entre le PER et les activités d'enseignement et d'apprentissage réalisées en classe. Par contre, ce sont ces dernières qui font référence pour construire un alignement avec les évaluations-bilans qu'ils conçoivent. Les enseignants expliquent que le PER est présent «dans leur tête » mais sans qu'ils ne s'y 
réfèrent matériellement. Ils consultent concrètement le plan d'études seulement en cas de doutes ou de dilemmes, par exemple s'ils doivent justifier une évaluation auprès d'un partenaire. Il apparaitt ainsi que l'usage du PER par les enseignants de notre recherche est indirect pour définir les contenus et les objectifs de leurs évaluations certificatives internes, ce qui représente un décalage avec les recommandations institutionnelles qui incitent les enseignants à utiliser le plan d'études comme référence première (voir ciaprès). Pour notre part, nous prenons très au sérieux le fait que ce sont les activités didactiques réellement effectuées avec les élèves qui font référence pour concevoir l'évaluation certificative, notamment parce que dans une perspective située de l'apprentissage (Brown, Collins \& Duguid, 1989), la forme, les moyens et les outils utilisés pour enseigner/apprendre font partie pleinement de ce que l'élève apprend, au même titre que les objets de savoir, et donc de ce qui sera aussi évalué. Un prochain enjeu est alors d'analyser finement la transposition didactique effectuée par les enseignants entre le prescrit (le plan d'études), les activités en classe (au regard aussi des moyens d'enseignement utilisés), les évaluations internes et externes.

\subsection{Entêtes de l'épreuve}

Dans le canton de Genève, une directive de l'enseignement primaire indique qu'un des principes pour construire tout contrôle écrit est d'utiliser un entête ou un fichet d'évaluation dans lequel les objectifs qui sont évalués doivent figurer, en des termes compréhensibles par les élèves. «Les objectifs sont à rechercher dans le plan d'études et à adapter au niveau de la formulation [...] Le fichet permet de collecter et de visibiliser clairement toutes les informations nécessaires à l'enseignant pour l'évaluation, à savoir : la date, le domaine ou l'axe thématique, la discipline, voire le titre de l'activité, les objectifs évalués, les observations de l'enseignant sur les démarches de l'élève et les conseils ou régulations proposées, ainsi que l'aide éventuelle de l'enseignant. » ${ }^{7}$. L'entête présente aussi la manière dont la note sera construite (seuils de réussite, barème,...) et offre un espace pour d'éventuels commentaires rédigés par l'enseignant en plus de la note chiffrée. La figure 2 illustre un entête issu de nos données du volet 1. 
Figure 2 : Exemple d'entête (volet 1)

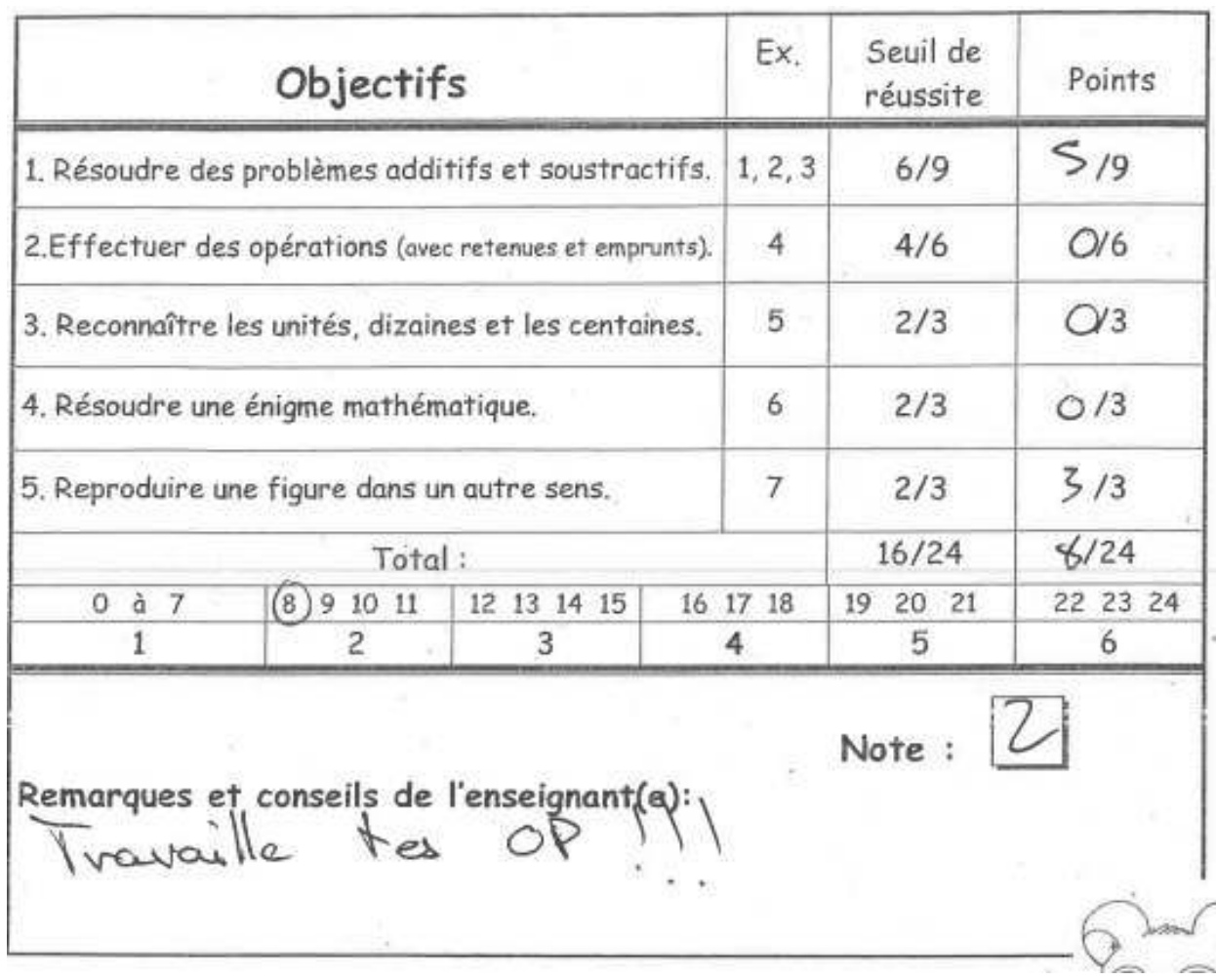

Nos premières analyses des contrôles écrits récoltés dans le volet 1 montrent une présence systématique des entêtes, conformément à des études précédentes qui ont montré que cette directive est devenue une norme dans les pratiques évaluatives des enseignants genevois (Mottier Lopez \& Tessaro, 2010). Toutefois, on observe des variations relativement importantes quant à leur contenu et à leur structuration. Les confrontations dans les diverses configurations sociales de la modération sociale ont fait ressortir que les enseignants s'accordent sur les aspects principaux suivants :

- indiquer précisément les objectifs et critères qui doivent être indépendants les uns des autres et pas trop nombreux;

- indiquer les points par objectifs / critères ;

- indiquer les exercices correspondants ;

- organiser les entêtes par composante ou domaine pour une bonne visibilité ;

- même si souvent le seuil de réussite est aux $2 / 3$ des points, ne pas le fixer de façon mécanique ;

- l'entête peut servir de liste de vérification pour les élèves lorsqu'ils sont dans une situation de production écrite, par exemple.

D'autres aspects, par contre, ont suscité des questionnements qui sont restés relativement ouverts, sans obtenir un consensus sur une "manière de faire" (Morrissette, 2011) :

- Faut-il formuler des indicateurs en plus des objectifs / critères, par exemple dans le correctif du contrôle écrit?

- Faut-il délimiter un seuil par composante / par domaine (regroupant plusieurs objectifs) plutôt que pour chaque objectif? 
- Comment trouver un juste équilibre entre une évaluation par objectifs spécifiques et une évaluation plus globale de la production de l'élève?

31 D'une façon générale, il ressort de notre analyse des savoirs collectifs négociés et validés par le groupe que ce sont essentiellement sur des aspects portant sur la construction de l'épreuve que les enseignants se sont mis d'accord en termes de pratiques communes et de comparabilité entre leurs évaluations certificatives internes. Pourtant, les modérations sociales portaient également sur les processus d'interprétation et de notation des productions des élèves. Ce niveau de jugement professionnel en évaluation est évidemment plus difficile à appréhender et à verbaliser que la construction des contrôles écrits. Mais ces premiers résultats tendent à montrer qu'il s'agit certainement d'un aspect de la pratique évaluative qui reste peu accessible à la conscience des enseignants ou qu'ils ne désirent pas forcément rendre publique. Poursuivons sur la présentation d'épisodes discursifs qui, précisément, montrent cette difficulté et la façon dont, à travers les échanges de modération, une distinction a progressivement émergé à propos des arrangements accomplis par les enseignants pendant les évaluations certificatives.

\section{De l'expérience située de la modération sociale à la formalisation d'une distinction conceptuelle}

\section{1. Émergence dialogique de significations autour de la notion d'arrangement évaluatif}

Les résultats présentés ici s'appuient sur une pré-analyse des échanges verbaux dans les différentes configurations de la modération sociale (débouchant sur une description narrative de la succession et de l'itération des thèmes abordés), puis sur la transcription et l'analyse des épisodes interactifs jugés significatifs par rapport à leur fonction épistémique. Un des thèmes abordés fut la question des arrangements évaluatifs, entendus d'abord comme des arrangements que l'enseignant s'autorise en cours de correction au sujet de l'attribution de points à un élève. Cette pratique est décrite dans la littérature (e.g. Merle, 2012) et attestée dans les pratiques que nous avons observées au cours du premier volet de notre recherche.

Lors de la première journée de modération sociale, il est apparu que les enseignants éprouvaient des difficultés à s'exprimer sur les arrangements qu'ils "s'autorisaient" dans leurs pratiques évaluatives. Pour la deuxième journée, nous avons donc sélectionné un extrait de verbalisation issue du volet 1 de notre recherche montrant un enseignant qui faisait un écart à son barème en le justifiant par la connaissance qu'il avait de son élève.

$\mathrm{Au}$ début de l'après-midi de la deuxième journée, les enseignants ont reçu une copie corrigée par un de leurs collègues et l'extrait en question. Le chercheur-modérateur a introduit la réflexion en ces termes : «On vous propose de vous interroger sur les informations que l'on utilise dans nos jugements qui ne relèvent pas directement de la production de l'élève. On a des informations sur l'élève, sur la culture de l'école, bref, des informations de tout ordre et qui, parfois, peuvent nous amener à prendre des décisions sur un point ou demi-point que l'on attribuera ou pas. On vous demande de vous mettre d'accord sur les informations qui selon vous semblent légitimes à pouvoir prendre en considération et les informations qui vous apparaissent totalement illégitimes ». À peine la consigne formulée, Coralie ${ }^{8}$ est intervenue : «mais c'est 
pendant l'élaboration du contrôle écrit ou pendant la correction?». Conformément au scénario envisagé par l'équipe de recherche et sur la base des constats issus de la première journée de modération sociale, il a été proposé aux enseignants de s'interroger plutôt sur les processus de correction. Un outil (sur le modèle de celui présenté dans le tableau 1) a été fourni aux trios d'enseignants afin qu'ils formalisent leurs accords et désaccords sur des catégories préexistantes (connaissances de l'élève, progrès, niveau de la classe, activités effectivement réalisées en classe, soutien apporté par la famille) et aussi des catégories ouvertes. Nous rapportons ci-dessous quelques extraits des échanges entre Coralie et Laurent (exceptionnellement en duo ce jour-là) puis de l'avancement des significations pendant la mise en commun qui ont contribué à l'émergence d'une conceptualisation renouvelée de la notion d'arrangement évaluatif.

Pendant les échanges en duo, Coralie exprime avec vigueur ses convictions: «Moi je prends en compte [la connaissance de l'élève] en élaborant les évaluations et en différenciant. Pas en corrigeant !... on peut le faire plus dans l'élaboration ou la passation de l'évaluation que dans la correction ». Coralie rejette en bloc l'idée d'arrangement en cours de correction. "Moi, éthiquement, ça me dérange ». La réflexion qui lui est demandée lui pose problème et l'interpelle: "Ou est-ce qu'on le fait inconsciemment?». Huit minutes plus tard, elle poursuit : "Mon élève non-francophone, je lui fais passer les évaluations comme les autres puis je la lui fais repasser systématiquement le lendemain. [...] Et systématiquement, il monte d'une "bonne" [...] Mais mon évaluation ne change pas. Quand je corrige, je corrige comme les autres". Clairement Coralie distingue ce qui relève d'un aménagement de son dispositif d'évaluation par rapport à certains élèves dans une visée de différenciation, de ce qui relève d'un arrangement en cours de correction. Dans les deux cas pourtant, un impact a lieu sur la note attribuée, mais seul le premier cas de figure lui parait légitime. Son collègue, Laurent, souligne que, selon lui, ce problème se posait moins quand il n'y avait pas l'obligation de «mettre des notes » mais sans argumenter. Les deux s'accordent finalement à contester la prise en compte d'informations autres que la stricte production de l'élève dans l'interprétation et appréciation de celle-ci. Ils rédigent leur prise de position dans l'outil de formalisation à leur disposition.

Puis le débat collectif commence, mettant en discussion les prises de position des différents groupes avec l'aide structurante du chercheur-modérateur. Coralie expose le point de vue de son duo. Quelques enseignants avancent qu'ils pratiquent parfois des arrangements en cours de correction. Une enseignante expose plus précisément les circonstances de ses arrangements, notamment quand la note est à la limite du seuil de réussite, dans des cas d'élèves très particuliers. Coralie écoute, intervient, résiste. Progressivement, il devient collectivement admis que les enseignants procèdent parfois à des arrangements mais qu'ils peinent à pouvoir les justifier : dans quelles situations cette pratique est-elle possible et légitime? Quels principes d'équité défendre dans ces cas ? Le chercheur-modérateur structure les échanges, se permet des incises théoriques. Il synthétise la pensée du collectif. "C'est très intéressant ce que vous dites car ceci n'est pas relevé dans la littérature. Il y aurait donc une distinction à faire entre des arrangements en cours de correction et des modifications en amont... ». Progressivement, une distinction de sens s'établit collectivement entre les interventions de l'enseignant qui servent à différencier le dispositif d'évaluation pour prendre en considération des situations particulières d'élèves et l'idée d'arrangement qui s'effectue pendant la correction de façon plus ou moins reconnue. Notons que cette distinction avait été pointée par Coralie dès sa première intervention ; une distinction qui, aussi, relève certainement de la structuration 
du dispositif de modération sociale avec les deux niveaux de négociation de consensus (conception ; appréciation).

\subsection{Ajustements du dispositif d'évaluation à des fins de régulation et de différenciation}

Le travail d'analyse ${ }^{10}$, que notre équipe de recherche a effectué a posteriori sur les savoirs collectifs négociés pendant les modérations sociales, amène à un renouvellement conceptuel de la notion d'arrangement. Nous distinguons désormais l'idée d'ajustement pour désigner les interventions effectuées par l'enseignant pour aménager délibérément son dispositif d'évaluation en fonction de la spécificité de la situation d'un ou de plusieurs de ses élèves, qui engage alors une modification des conditions sociales, matérielles, symboliques des pratiques évaluatives. Ainsi, la notion d'ajustement désigne une fonction assumée, voire revendiquée par les praticiens, de régulation et de différenciation des situations d'évaluation, y compris certificatives.

Trois grandes catégories d'ajustements peuvent être délimitées en fonction de la temporalité de l'évaluation certificative instrumentée par un contrôle écrit :

- En amont du contrôle écrit, par exemple pour assurer un alignement entre activités didactiques et évaluatives, pour prendre en considération le niveau du groupe classe, pour harmoniser une pratique établie dans un établissement scolaire donné ;

- Pendant le contrôle écrit, par exemple en effectuant si nécessaire des relances individuelles ou collectives (reformuler la consigne, expliquer un mot, donner un synonyme, ...), en aidant un élève à entrer dans la tâche, en laissant du temps supplémentaire à un élève pour terminer son évaluation;

- Après la passation du contrôle écrit, par exemple en rédigeant des commentaires ciblés et différenciés, en consultant des collègues ou des spécialistes pour les élèves ayant des troubles spécifiques d'apprentissages.

À noter que les exemples rapportés ici sont ceux qui ont fait l'objet d'un accord partagé ou admis entre les 18 enseignants de notre recherche. Ainsi, la notion d'ajustement permet aux enseignants de penser l'évaluation certificative dans une visée éducative, rendant légitimes certaines différenciations et l'éloignant d'une logique standardisée, telle celle des épreuves externes. Au plan théorique, cette notion permet de contribuer à une modélisation plus intégrative des fonctions formative et certificative des évaluations situées et internes des enseignants.

\subsection{Arrangements évaluatifs pendant la correction}

Quant à la notion d'arrangement évaluatif (au sens de Merle, 2012), nous proposons de la réserver aux compromis que l'enseignant effectue plus ou moins consciemment par rapport à des contraintes interpersonnelles et/ou institutionnelles, notamment pendant le processus d'interprétation et de prise de décision en cours de correction des productions des élèves. Ces arrangements peuvent être vus comme fonctionnels, mais ils sont rarement assumés par les enseignants qui semblent les accomplir à défaut de ne pas pouvoir/savoir faire autrement. Les arrangements évaluatifs relèvent donc plutôt d'aspects peu assumés, voire clandestins, du travail évaluatif de l'enseignant. 
41 Dans notre recherche, des débats nourris ont eu lieu sur la prise en compte, dans le processus de jugement évaluatif, des connaissances que l'enseignant possède de l'élève, par exemple au regard de son profil scolaire, des activités réalisées en classe, des progrès effectués, de sa biographie notamment. Clairement, les enseignants sont sensibles à l'effet d'assimilation (Noizet \& Caverni, 1983) ou de stéréotypie que ces connaissances sont susceptibles de produire sur leurs évaluations. Ces comportements ont pourtant été observés dans le volet 1 de la recherche et, parfois, verbalisés par les enseignants dans l'entretien semi-directif associé à l'observation de leur jugement-en-acte. Mais pendant les modérations sociales, soit les enseignants n'ont pas conscience qu'ils effectuent ce type d'arrangements en cours d'évaluation, soit ils peinent à l'admettre socialement pour des questions d'équité plus difficiles à justifier que les ajustements réalisés sur le dispositif d'évaluation dans une perspective de prise en compte des spécificités des élèves et des situations.

\section{En guise de conclusion provisoire}

42 De manière cohérente avec notre positionnement épistémologique situé, le dispositif de modération sociale semble bel et bien un lieu privilégié non seulement de négociation de savoirs collectifs mais également propice à l'émergence de savoirs nouveaux dans un processus de sémiose qui se poursuit potentiellement par la suite. Cette "propriété » est d'un intérêt majeur pour la formation mais aussi pour la recherche. En effet, cette construction/négociation concerne tout autant les enseignants que les chercheurs. Les premières analyses montrent clairement (et là encore en toute cohérence avec l'hypothèse située) que ce processus social se joue dans une dialectique où l'acteur individuel (qu'il soit chercheur ou enseignant) permet un avancement des savoirs collectifs en même temps que le collectif permet un développement de la pensée individuelle.

43 Un ensemble d'analyses de nos données restent à effectuer, notamment pour mettre en relation les trois volets de notre recherche ce qui, peut-être, nous permettra d'observer quelques traces d'un développement professionnel chez les enseignants, notamment dans la conception de leurs contrôles écrits et dans la verbalisation de leurs jugements-enacte. De plus, nous répliquons actuellement l'entier de notre dispositif avec des enseignants de 7P-8P HarmoS (élèves de 10-12 ans), afin d'effectuer une étude longitudinale sur les 4 années de la fin de l'école primaire qui amènent aux enjeux de l'orientation vers les filières de l'enseignement secondaire. Les défis sont à nos yeux importants, tant en termes de connaissances scientifiques sur les pratiques d'évaluation certificative individuelle et dans une perspective collaborative, qu'en termes d'ingénierie pour penser des dispositifs de formation initiale et continue des enseignants. 


\section{BIBLIOGRAPHIE}

Allal, L. (2009). Pratiques évaluatives des enseignants face aux méthodologies des recherches évaluatives portant sur les systèmes scolaires. In L. Mottier Lopez \& M. Crahay (Ed.), Évaluations en tension : entre la régulation des apprentissages et le pilotage des systèmes (pp. 29-45). Bruxelles : De Boeck.

Allal, L., \& Mottier Lopez, L. (à paraître). Teachers' professional judgment in the context of collaborative assessment practice. In C. Wyatt-Smith \& V. Klenowski (Ed.), The enabling power of assessment. London: Springer.

Brown, J. S., Collins, A., \& Duguid, P. (1989). Situated cognition and the culture of learning. Educational Researcher, 18(1), 32-42.

Butera, F., Buchs, C., \& Darnon, C. (Ed.). (2011). L'évaluation : une menace? Paris : Presses Universitaires de France.

Dechamboux, L. (2012). Étude exploratoire : les Figures de Jugement, essai d'analyse des processus de jugement évaluatif des enseignants. Mémoire de maîtrise. Université de Genève.

Gilliéron Giroud, P., \& Ntamakiliro, L. (Ed.). (2010). Réformer l'évaluation scolaire : mission impossible ? Berne : Peter Lang.

Harlen, W. (2007). Assessment of learning. London: SAGE.

Lave, J. (1988). Cognition in practice: Mind, mathematics and culture in everyday life. Cambridge, MA: Cambridge University Press.

Laveault, D. (2009). L'amélioration de l'efficacité du système éducatif : sur quels indicateurs s'appuyer? In X. Dumay \& V. Dupriez (Ed.), L'efficacité dans l'enseignement : promesses et zones d'ombre (pp. 177-194). Bruxelles: De Boeck.

Linn, R. L. (1993). Linking results of distinct assessments. Applied Measurement in Education, 6(1), 83-102.

Maxwell, G. (2002). Moderation of Teacher Judgments in Student Assessment. Brisbane : Queensland School Curriculum Council.

Merle. P. (2012). L'évaluation des élèves. Une modélisation interactionniste des pratiques professorales. In L. Mottier Lopez \& G. Figari (Ed.), Modélisations de l'évaluation en éducation (pp. 27-43). Bruxelles : De Boeck (collection Raisons Éducatives).

Morrissette, J. (2011). Vers un cadre d'analyse interactionniste des pratiques professionnelles. Recherches qualitatives, 30(1), 38-59.

Morrissette, J., Mottier Lopez, L., \& Tessaro, W. (2012). La production de savoirs négociés dans deux recherches collaboratives sur l'évaluation formative (pp. 27-43). In L. Mottier Lopez \& G. Figari (Ed.), Modélisations de l'évaluation en éducation. Bruxelles : De Boeck (collection Raisons Éducatives).

Mottier Lopez, L. (sous presse). Vers une culture de l'évaluation conjointement construite. In J.-F. Marcel \& H. Savy (Ed.), Évaluons, évoluons. De l'évaluation dans l'Enseignement agricole. Dijon : Educagri Editions Recherche. 
Mottier Lopez, L. (2012). Interprétation d'une recherche collaborative dans les termes de la «valuation » de Dewey. Travail et apprentissage, 183-199.

Mottier Lopez, L., \& Laveault, D. (2008). L'évaluation des apprentissages en contexte scolaire : développements, enjeux et controverses. Mesure et évaluation en éducation, 3, 5-34.

Mottier Lopez, L., \& Tessaro, W. (2010). Evaluation des compétences à l'école primaire genevoise : entre prescriptions et pratiques. Mesure et évaluation en éducation, 33 (3), 29-53.

Noizet, G., \& Caverni, J.-P. (1983). Les procédures d'évaluation ont-elles leur part de responsabilité dans l'échec scolaire ? Revue française de pédagogie, 62, 7-14.

Pourtois, J.-P. \& Desmet, H. (1997). Epistémologie et instrumentation en sciences humaines (2 è éd.). Sprimont : Maradaga.

Weber, M. (1919). Le savant et le politique. Version numérique dans le cadre de la collection « Les classiques des sciences sociales ». Consulté le 2.10.2012 sur :

http://classiques.uqac.ca/classiques/Weber/savant_politique/Le_savant.pdf

Wenger, W. (1998). Communities of practice. Learning, meaning, and identities. Cambridge, UK: Cambridge University Press.

Wyatt-Smith, C., Klenowski, V., \& Gunn, S. (2010). The centrality of teachers' judgement practice in assessment: a study of standards in moderation. Assessment in Education : Principles, Policy \& Practice, 17(1), 59-75.

\section{NOTES}

1. Lave (1988) parle d'arena (dimension pré-existante) et de setting (dimension émergente) du contexte.

2. Le terme "contrôle écrit » désigne tout instrument d'évaluation composé d'items écrits (questions, consignes, problèmes, etc.) auxquels l'élève doit répondre par écrit.

3. Voir la table de conversion des degrés scolaire selon HarmoS : http://icp.ge.ch/dip/ecdip/ spip.php?article19

4. Depuis, notre équipe s'est agrandie avec l'arrivée de Sophie Serry. Nous la remercions pour sa lecture attentive de l'article.

5. Voir : http://www.plandetudes.ch/web/guest/per

6. Ou, par cas de force majeure, en duo.

7. http://petit-bazar.unige.ch/system/files/activite-bilan-CM-v2.pdf

8. Prénom d'emprunt.

9. Expression genevoise qui désigne la note. Si par exemple l'élève a un 3 sur 6 lors de la première passation, cela signifie qu'il obtiendra un 4 à la deuxième passation.

10. Dans une approche inductive-déductive qui caractérise notre ancrage épistémologique. 


\section{RÉSUMÉS}

L'article présente une recherche sur des pratiques d'évaluation certificative des apprentissages à l'école primaire genevoise. La recherche articule un dispositif de développement professionnel des enseignants et des démarches plus classiques de recherche qualitative. L'article présente le dispositif mis en place pour favoriser des pratiques de «modération sociale » entre enseignants et expose les principaux savoirs collectifs négociés par les participants, en termes, d'une part, de pratiques d'évaluation certificative vues comme devant être communes entre les classes et, d'autre part, de différenciation possible de l'évaluation. Il montre comment, dans les échanges intersubjectifs, une distinction conceptuelle a émergé entre les notions d'ajustement des dispositifs de l'évaluation et d'arrangement évaluatif souvent effectué inconsciemment pendant que l'enseignant corrige les copies de ses élèves. Quelques enjeux de la construction d'une culture partagée de l'évaluation sont discutés.

The article presents a research on practices of certificative assessment in primary school in the canton of Geneva. The research is aimed at sustaining teachers' professional development and is structured around classic design of qualitative studies. The article describes a device designed to favour practices of "social moderation" among teachers and exposes the main collective knowledge negotiated by the participants: on one hand about the aspects of the assessment practices that should be common between the classrooms, on the other hand, about the possible differentiation of those practices. It shows how a conceptual distinction has emerged during the intersubjective exchanges with regard to the notions of adjustment of the assessment devices and of the evaluative arrangement that are often unconsciously made during the correction of the students' copies. Some issues concerning out of the construction of a shared culture of assessment are discussed.

\section{INDEX}

Mots-clés : évaluation certificative, modération sociale, culture de l'évaluation, ajustements, arrangements évaluatifs

Keywords : certificative assessment, social moderation, culture of assessment, adjustment, evaluative arrangement

\section{AUTEURS}

\section{LUCIE MOTTIER LOPEZ}

Université de Genève

WALTHER TESSARO

Université de Genève 
LIONEL DECHAMBOUX

Université de Genève

\section{FERNANDO MORALES VILLABONA}

Université de Genève 